\title{
GERENCIA DE PORTAFOLIOS FINANCIEROS INTERNACIONALES
}

\section{MANAGEMENT OF INTERNATIONAL PORTFOLIO FINANCIAL}

\author{
Nicko Gomero Gonzales ${ }^{1}$ \\ Docente Asociado de la Facultad de Ciencias Contables, UNMSM
}

(Recepción: Setiembre de 2009 / Conformidad: Octubre de 2009)

\section{RESUMEN}

Si los mercados financieros se volatilizan, los agentes económicos deben de evaluar nuevas alternativas para colocar sus capitales de riesgo. En la Zona del Euro, Asia como Norteamérica, se desarrollan mercados bursátiles que dan la oportunidad de demandar títulos de renta variable de las empresas globales más importantes del planeta, como Chevron, Walt Disney, Telefónica, Yahoo, Mac Donalds. Como dicen los manuales financieros, la mejor estrategia para minimizar los riesgos por inversión de esta naturaleza, es estructurando una cartera bien diversificada, a la luz de esta teoría entonces, sería preciso que los inversionistas coberturadores o especuladores, sean institucionales o particulares, gerencien títulos de los mercados bursátiles globales, que como se sabe están representados por el Hang Seng, IBEX, Nasdaq, Dow Jones, Bobespa, entre otros, solo así estará en una posición ventajosa para contrarrestar los choques derivados de los ciclos económicos.

Palabras claves: Mercados financieros, volatilización, capital de riesgo, coberturadores, especuladores, mercados globales, ciclo económico

\begin{abstract}
If financial markets are volatilized, the traders have to evaluate new alternatives to place their capital at risk. In the Euro Area, Asia and North America, stock markets are developed which provide the opportunity to demand equity securities of major global corporations on the planet, like Chevron, Walt Disney, Telephonic, Yahoo, Mac Donald's. As financial manuals say, the best strategy to minimize risks for investment of this nature, is structuring a well diversified portfolio, in light of this theory then, would need to hedge or speculative investors, whether institutional or retail, management titles global stock markets, which as you know are represented by the Hang Seng, FTSE, Nasdaq, Dow Jones, Bobespa, among others, only then will be in an advantageous position to counter the shocks stemming from economic cycles.
\end{abstract}

Keywords: Financial Markets, volatilization, venture capital, hedge, speculators, global markets, business cycle

1 Doctor en Ciencias Económicas. Docente de la Unidad de Postgrado de la Facultad de Ciencias Contables, UNMSM. E-mail: nickgo_6@msn.com 


\section{OBJETIVo GENERAL DEL ESTUDIO:}

Evaluar el Mercado bursátil global a fin de direccionar inversiones de riesgo

\section{OBjETIVOS ESPECífICOS}

- Evaluar el mercado bursátil de la zona del Euro

- Evaluar el mercado bursátil del mercado norteamericano, representado por el Dow Jones.

\section{MARCO GENERAL}

La crisis financiera internacional exige mirar otros mercados y a la vez buscar nuevas opciones de inversión. Si bien muchos inversionistas, particulares o institucionales, se muestran temerosos de penetrar en mercados internacionales, pero otros, más apegados al riesgo, no sudan en demandar activos financieros, que dependiendo de la calidad de los mismos, le reportará importantes rentabilidades o en todo caso le permitirá estar mejor cubierto ante choque cíclicos de los mercados financieros. Tal como señala Markowitz en la Teoría del Portafolio, cuando más títulos tenga un inversionista tenderá a bajar sus niveles de riesgo, específicamente, el diversificable, situación que le permitirá al tenedor de los títulos estar en mejor posición para gerenciar su portafolio. Hay que recordar que solo el riesgo único se puede minimizar con la diversificación, y no asa el riesgo de mercado, el cual se va a presentar de igual manera y en la misma magnitud si está o no coberturado. Es preciso señalar que Harry Max Markowitz fue un Economista estadounidense especializado en el análisis de inversiones. Recibió el Premio Nobel de Economía en 1990 junto a Merton Miller y William Sharpe por sus aportaciones al análisis de carteras de inversión y a los métodos de financiación corporativa.

La actual crisis financiera internacional, que según parece no podrá ser revertido ni con políticas agresivas de tipo monetaria y fiscal, una clara señal de lo afirmado es que el desempleo en las principales economías del mundo se sigue profundizando, tal es así que en Estados Unidos ya bordea el 9.7\% y en España el 19\%, es más el FMI sigue ajustando las tasas de crecimiento económico en estas economías con cifras negativas, ver el grafico $\mathrm{N}^{\circ} 1$, donde se observa claramente que solo la China tendría un crecimiento importante pero por debajo de los experimentado en años anteriores. (Ver grafico $\mathrm{N}^{\circ} 1$ )

\section{Graf. $\mathrm{N}^{\circ}$ 1.Ajustes realizados por el $\mathrm{FMI}$ sobre el crecimiento del $\mathrm{PBI}$ en las principales economías del mundo: 2009}

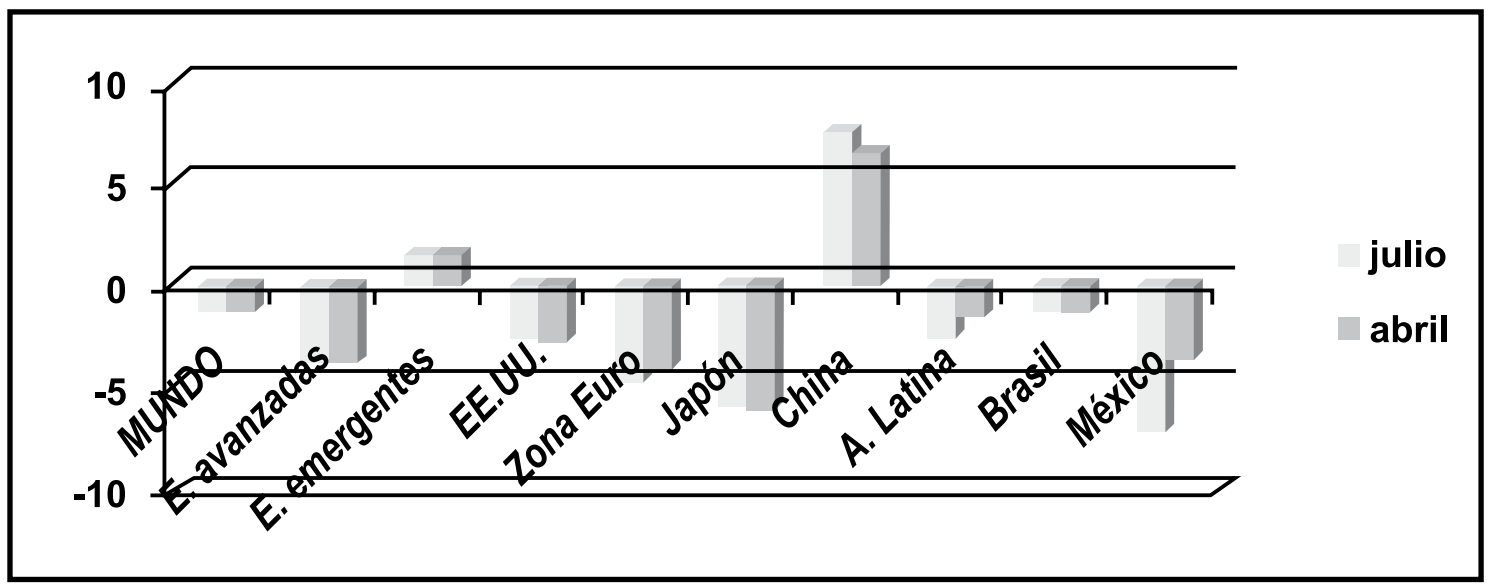

Fuente: FMI

Elaboración propia

El escenario que proyecta el FMI para la economía mundial no es tan optimista, todo lo contario nos dan una lectura de serio entrampamiento que, por las características presentadas se puede afirmar que los factores que han originado estas perturbaciones económicas y financieras son de carácter estructural, mejor dicho, son fallas del propio modelo del libre mercado, lo cual exigirá una mayor cantidad de tiempo de lo pronosticado por el FMI para salir de la crisis global.

Como argumentan algunos analistas económicos, la filosofía del «laissez faire, laissez passer», enmarcado en la globalización de los marcados ha dejado de tener vigencia, dado que en el mundo se viene presentando mayor asimetría económica y social, justamente como producto de la aplicación 
de esta doctrina económica. La crisis económica ha empujado a muchos gobiernos a participar en la economía realizando salvatajes financieros a grandes bancos globales y empresas de la misma naturaleza. Esta nueva posición política hace pensar que también han entendido que el libre mercado no conduce a lo que realimente exige la sociedad global. Quizás los especuladores, sean quienes no se desprendan de la filosofía del mercantilismo para acumular más riqueza, mas no es lo que necesita actualmente para articular estructuralmente las economías del mundo.

Si bien los principios filosóficos del libre mercado y la globalización han conducido que muchas economías del planeta logren avanzar en su frente externo e interno, tal como es el caso de la China, la India, Brasil, pero a la misma vez este mismo modelo de gestión económica ha creado espacios de vulnerabilidad que justamente ahora se ha traducido en crisis global. Las brechas de fragilidad que se han originado en el mundo no solo es de tipo financiero, sino que existen fenómenos sociales y ambientales muchos más peligrosos, ejemplo de ello es la exclusión y las asimetrías sociales, que de no darse solución en forma integral en el corto o mediano plazo, en un futuro cualquier intento para estabilizar los mercados tenderían a débiles resultados y en el peor de los casos serian totalmente estériles.

Bajo un nuevo modelo de gestión económica no seria suficiente que cada país, especialmente aquellas consideras las más ricas e industrializadas del mundo, como son Estados Unidos, países de la Zona del Euro y del Continente Asiático, traten de dar solución a sus problemas unilateralmente. Las políticas públicas que puedan ensayar sus respectivos gobiernos respectivos tienen que tener una visión global e integradora y a la vez poseer una alta dosis de contenido social.

Cuando los gobiernos, en especial aquellos que poseen el liderazgo del mundo, mueven una ficha dentro del tablero económico para resolver turbulencias de sus mercados, los efectos de dichas medidas no solo se focalizaran en su frente interno, sino dada las características de la globalización económica traspasaran sus fronteras, donde se localizan países que al final de cuentas terminan por asumir los costos de la crisis. Bajo este contexto, el escenario económico actual amerita que las políticas de carácter estratégica aparte de ser sincronizadas y coordinas a nivel de las principales economías del mundo deben estar encuadradas bajo la filosofía de la responsabilidad social.

$\mathrm{Al}$ margen de las de la deficiencias estructurales que presenta el actual modelo económico, en un mundo globalizado, donde la información corre a la «velocidad de la luz», los efectos de cualquier perturbación económica también se globalizan, ya sean negativos o positivos, por ello los agentes económicos deben estar mejor preparados para lograr espacios de mercado que les reporten resultados ventajosos o en todo caso logren minimizar pérdidas por operación, que también es otra forma de optimizar operaciones financieras. Ante escenarios de turbulencia económica, la inteligencia financiera debe conducir al inversionistas a optar por diversas alternativas de inversión, pudiendo ser estas de mayor o menor riesgo, para ello están los mercado globales, que sin bien es cierto están atravesando por graves dificultades financieras, pero no dejan de ser un camino critico para lograr mayores expectativas de rendimiento

Tal como ya se señalo, cuando la economía domestica no reporta oportunidades para diversificar portafolios, queda la opción del mercado de ultramar, es así que el inversionista puede acudir a la bolsa de Tokio, de Singapur, Estados Unidos, España, etc. Mercados en la que convergen capitales y valores de diversa naturaleza, no solo acciones sino también Derivados de productos financieros y reales, que genera posiciones ventajosas para inversionistas especulativos o coberturadores. A continuación describiremos los mercados bursátiles más importantes del mundo, señalando sus características y que ofrecen para colocar los capitales de inversión.

\section{A. EUROPA}

El índice IBEX 35 (Iberia Index) es el principal índice de referencia de la Bolsa Española. Está formado por las 35 empresas con más liquidez que cotizan en el Sistema Interconexión Bursátil Electrónico (SIBE) en las cuatro Bolsas Españolas (Madrid, Barcelona, Bilbao y Valencia).

E1 Eurostoxx 50 : es el índice bursátil de referencia que emula el comportamiento de los precios de las acciones de las 50 empresas más grandes de la zona euro. Estas acciones son blue chip, término que hace referencia a las acciones más importantes de las bolsas de valores, que poseen la mayor capitalización de mercado y un alto valor cualitativo.

El CAC 40 (Cotation Assistée en Continu), que toma su nombre del primer sistema de automatización de la Bolsa de Paris, es un Índice Bursátil Francés, una referencia para el Euronext Paris. este índice es una medida ponderada según la capitalización de los 40 valores más significativos de entre las 100 mayores empresas negociadas en la Bolsa de París. 
El índice DAX o Xetra DAX (Deutscher Aktienindex) es el Índice Bursátil referencia de la Frankfurter Wertpapierbörse (Bolsa de Francfort) Se le conoce también como Dax 30. Es el índice más conocido de la Bolsa alemana. Su cálculo consiste en una media aritmética ponderada por capitalización

\section{ASIA}

Nikkei 225 (, Nihon Heikin Kabuka), comúnmente denominado Indice Nikkei, es el más popular del mercado japonés, lo componen los 225 valores más líquidos que cotizan en la Bolsa de Tokio. Estos valores se caracterizan por su elevada liquidez

Hang Seng Index: es el principal índice bursátil chino de Hong Kong. Es usado para grabar y monitorizar diariamente los cambios de las más grandes compañías de Hong Kong en el mercado de acciones. Consiste en 33 compañías representando el 65\% de Hong Kong Stock Exchange. Sus valores ponderan por el criterio de capitalización. Para formar parte del índice el valor debe estar dentro del $90 \%$ de empresas con mayor capitalización y volumen y haber cotizado en la Bolsa de Hong Kong durante más de 24 meses.

\section{B. AMERICA}

El Índice bursátil Dow Jones es un servicio de información financiera que muestra un conjunto de diferentes índices bursátiles de los mercados de EstadosUnidos calculados por la empresa Dow Jones \& Company. Por su importancia a veces se habla como índice Dow Jones sin especificar al referirse al más importante de ellos que es el industrial (DJIA), pero es una forma incorrecta de expresarlo, ya que como índice Dow Jones existen una diversidad de ellos

Nasdaq 100 Index (Índice Nasdaq 100) conocido también como simplemente Nasdaq $\mathbf{1 0 0}$ es un índice bursátil de Estados Unidos quee recoge a los 100 valores de las compañías más importantes del sector de la industria incluyendo empresas de hardware y de software, las telecomunicaciones, venta al comercio al por menor/por mayor y biotecnología inscritos en la Bolsa de Nueva York( NYSE), listadas en el NasdaqStock Market. En el índice pueden estar tanto empresas americanas como internacionales.El principal indicador del Nasdaq 100 es el Nasdaq 100 Index. Este índice no incluye valores financieros incluyendo las compañías de inversión lo que lo diferencia de otros índices como el S\&P500

La Bovespa es hoy una de las mayores bolsas de valores del mundo, situada en el corazón de la mayor ciudad brasileña, Sao Paulo. Como media, se intercambian acciones por valor de1.221,3 millones de reales cada día (según datos de 2004)).

Merval .Es el principal índice del Mercado de Valores de Buenos Aires. Este índice muestra la evolución en conjunto de las 11 empresas argentinas más representativas del mercado. Es modificado cada 3 meses. Cada acción tiene un peso o ponderación con respecto al índice y esa ponderación se determina con respecto al volumen negociado

Una rápida mirada a la dirección electrónica de www.finazas.com. Nos abre una abanico de oportunidades para demandar un activo financiero de nuestro interés tal como es : Yahoo, Amazon. com, Aplle.Inc, Adobe System, entro otros . Si su preferencia es el Dow Jones, aquí algunas opciones de inversiones :Alcoa Inc, Bank of Amer, Chevron Corp, J P Morgan, Walt Disney .

Si quiere incursionar en la bolsa española, también hay interesantes empresas, como Telefónica , Abengoa, Bbva,Mafre, Repsol Ypf.

Un portafolio tentativo que podría ensayar un inversionistas es comprando acciones en España (IBEX) , Estados Unidos ( Nasdaq) y en Sao Paulo (Bovespa), si el mercado español sufre un revés , estaría protegiéndose con los resultados de los otros mercados bursátiles, claro está dependiendo de cómo haga la diversificación, mejor dicho como se de la covariabilidad de los activos financieros.

Como se puede apreciar, las oportunidades de inversión están dadas están dadas, solo tiene que acertar en el blanco para que salga ganado de la posición donde se encuentra

Asimismo se recomienda realizar un análisis técnico y fundamental para tomar la mejor decisión, pero no hay que dejar de lado los estadígrafos, como la desviación típica, el coeficiente de correlación, el Beta, la covarianza, que son instrumentos esenciales que conducirán a lograr mejores resultados en una cartera diversificada.

$\mathrm{El}$ análisis fundamental se basa en el estudio minucioso de los estados contables de una empresa así como de sus expectativas futuras, planes de expansión y factores del entorno socioeconómico que puedan afectar a la marcha de la empresa. Para alcanzar una valoración sobre un valor, el analista realiza estimaciones de ventas, cash flows, beneficios y dividendos futuros. El analista fundamental fija precios objetivo basados en descuentos de cash flows futuros, niveles de PER, crecimientos de beneficios, comparaciones con el mercado y el sector y suma de partes de los distintos negocios (entre otros métodos) y le asigna recomendaciones de sobreponderar (si piensa que el valor se comportará mejor que el mercado), neutro (si cree que se comportará 
como el mercado) e infraponderar (si estima que su comportamiento será peor que el mercado).

El Análisis Técnico es el que se utiliza para interpretar un gráfico y permite ver qué es lo que sucede al instante y actuar en consecuencia. Examina datos pasados sobre cotizaciones y volúmenes negociados, con el objetivo de prever futuros movimientos de precios. Estudiando los gráficos con herramientas específicas, se descubren tendencias (primarias o secundarias) y se identifican oportunidades de compra o de venta. Los gráficos pueden ser anuales, semanales o diarios. Para las inversiones diarias («day trading»), el análisis se puede centrar en gráficos con parámetros incluso menores, como son: gráficos de 1 hora, de media hora, de 5 minutos y en algunos casos se llega hasta el análisis de los gráficos de 1 minuto. El objetivo de estos análisis es saber cuándo comprar y cuándo vender.

Aquí un ejemplo de análisis fundamental para las acciones globales de la telefónica, que negocia en el IBEX
Últimos resultados. 13/05/2009. El beneficio neto de Telefónica subió en el primer trimestre de 2009 un 9,8 por ciento hasta 1.690 millones de euros. Los ingresos, frenados por la caída de tarifas, la evolución cambiaria adversa y la recesión en algunos de sus principales mercados, bajaron un 1,4 por ciento a 13.703 millones de euros. El impacto negativo de los tipos de cambio restó 4,7 puntos porcentuales al crecimiento de los ingresos.

El resultado operativo antes de intereses y amortizaciones OIBDA cedió un 0,4 por ciento a 5.376 millones de euros. La compañía dijo en febrero que espera para 2009 un aumento interanual del OIBDA consolidado en el rango de $+1 \% /+3 \%$.

En términos orgánicos, el resultado bruto de explotación creció un 2,5 por ciento en el primer trimestre del 2009.

\begin{tabular}{|c|c|c|c|c|c|}
\hline Г.I INI:I & Aห่แ วกก5 & คพ่I วกกร & Añ 1 >nn? & ดับ ว วกค & 4 \\
\hline "Dliakgul & $\varepsilon 7 . \varepsilon=2.1 \cdot 5$ & $52.963 .5 \div$ & $50 \therefore \mid c, 57 \xi$ & 57.915 .756 & $2, \overline{8}=$ \\
\hline FAn & דור:... & F.TF & n is․‥ I5: & In. П।5. & $\therefore, 1>2$ \\
\hline Кг. $\Gamma^{4}$ & 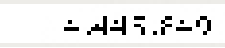 & f.7:5.9:5 & 21 6.5.: & T.RᄀK.R.5? & $1-, 1>0$ \\
\hline L'af alizacic ${ }^{2}$ & L2.'4j.':3 & & 144 1's.60: & $t 4.3 t 4.1 \mathrm{US}$ & $-204=$ \\
\hline ic: snes $s^{3}$ & $\angle . \subsetneq 21.130$ & $4.9 \div 1 .=3$ & $477:-49$ & 4.704 .996 & $-1,44^{\circ}=$ \\
\hline 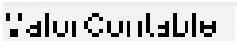 & ここ̇158116 & 20031053 & $Z 285 \cdot 88 \mathrm{r}$ & $17231 \cdot 05$ & $-\hat{L}^{*}, \bar{c} 1 \bar{x}_{2}$ \\
\hline 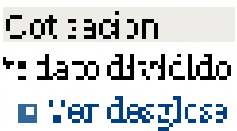 & $\begin{array}{l}1 \geq . \bar{r}- \\
1 \mathrm{Jc} \\
\text { re:لدadcs }\end{array}$ & 15,12 & $21.8 z$ & $15, \mathrm{a} 5$ & $-2 \bar{r}, z=\bar{z}$ \\
\hline
\end{tabular}

\section{Análiss}

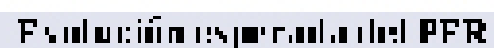

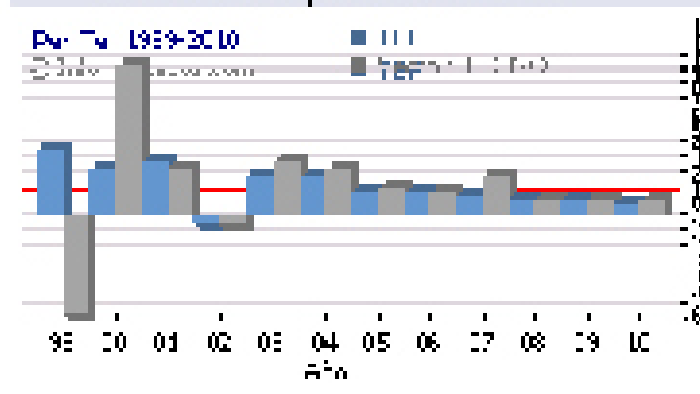

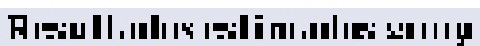
Chifi: NeqDCID: BDI:

Lash I lom:

Dividendos:

-atcs diricicss erJ: 14 Ju $\therefore<<0$ JUU

$75^{2} \cdots 1$

$10:$ :

$5+10 \div \div$
26/06/2009. Cuenta con uno de los mejores potenciales de crecimiento a largo plazo en Europa, dispone de una situación financiera saneada y buena retribución al accionista. En 2009 ha dicho qeu repartirá $1.5 € /$ acción, lo que supone una rentabilidad sobre dividendo del $9.5 \%$ y se ha comprometido a aumentar progresivamente la retribución al accionista en próximos ejercicios.

Compró la compañía británica de telefonía móvil O2, segundo operador de móviles en Reino Unido y tercero en Alemania, por una cifra de 26.283,7 millones de euros. Un precio elevado pero los fuertes 
objetivo de crecimiento de $\mathrm{O} 2$ justifican la operación. Esta adquisición supone una reorientación de la estrategia de Telefónica; hasta ahora, la operadora se había centrado, principalmente, en reforzar su presencia en el mercado latinoamericano. Sin embargo, con O2, entra de lleno en el mercado europeo y apuesta por el negocio de telefonía móvil (al reducir el peso de la telefonía fija en sus cuentas). Además diversifica el origen geográfico de sus ingresos.

Por otra parte, la venta de su filial Endemol le ha reportado ingresos que compensan la fuerte inversión en la compra de la británica.

El fuerte crecimiento de Latinoamérica y la excelente resistencia de la actividad en España siguen siendo los pilares del éxito de Telefónica. En su mercado nacional, el aumento de las ventas y de la rentabilidad es la envidia de la mayoría de los grandes operadores nacionales europeos. Destaca asimismo su buen hacer en móviles, dada la reciente llegada de nuevos competidores.

En contra: guerra de precios, y tendrá que hacer frente a un futuro endurecimiento reglamentario (bajada de tarifas de llamadas en Europa). En cambio, su actividad en Alemania y el Reino Unido han decepcionado algo. Latinoamérica sigue siendo una zona de inversión prometedora para el grupo.

Pese al considerable incremento de la deuda en los últimos años tras las importantes adquisiciones, la elevada liquidez generada por sus actividades le permite poner coto a la deuda, aprovechar nuevas oportunidades de inversión y remunerar correctamente al accionista. Para el ejercicio en curso, la compañía se ha fijado el objetivo de elevar sus ingresos entre un $6 \%$ y un $8 \%$, (lo que supondría ingresar 60.000 millones de $€$ ) el Oibda entre un $7,5 \%$ y un $11 \%$, y el resultado operativo en un rango de entre el $13 \%$ y el $19 \%$. Además, la cifra anual de inversión se situará en torno a los 8.600 millones de euros.

Cotiza a 9.5 veces beneficios estimados, frente a una media sectorial de $10.3 \mathrm{v}$ y un ratio histórico para la operadora de $14.2 \mathrm{v}$. Yield del 7\%, frente a una rentabilidad del $7.4 \%$ para el Ibex y una media del 5.3\% para las compañías del EuroStoxx 50. Le asignamos potencial a $19 € /$ acción a medio/largo plazo.

Si se quiere hacer un análisis técnico, se presenta el caso del IBEX (mercado bursátil español)

\section{Gráfico N`2. Comportamiento del IBEX: 2009}

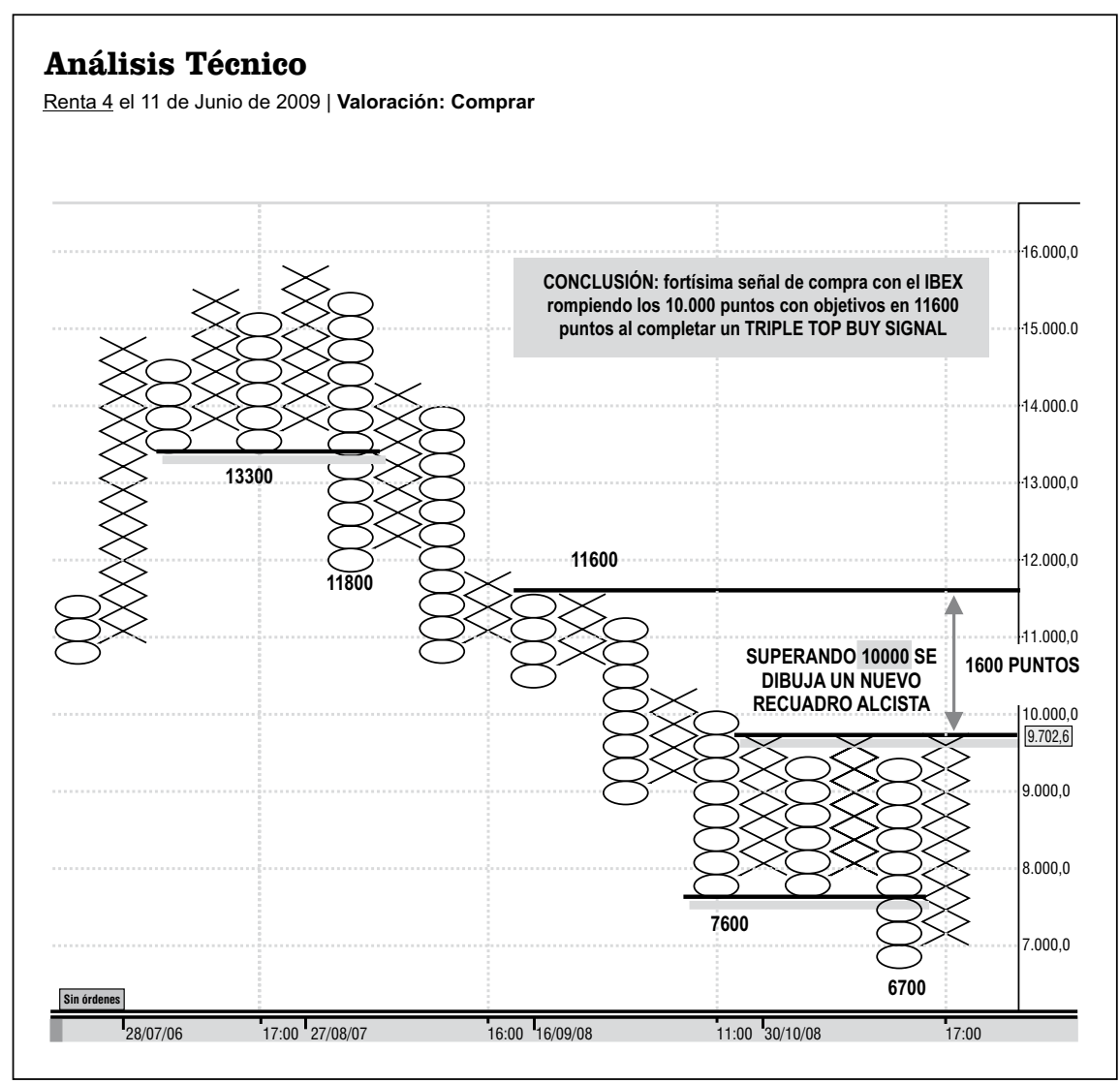


IBEX 35. Los gráficos de punto y figura permiten centrase en el precio, ignorando el ruido del mercado, por lo que las determinadas señales, como se pude ver, tienen altos grados de fiabilidad para el mediano plazo.. cuando el precio 300 puntos se dibuja con una «X» y cuando baja se dibuja con una «O». como se puede apreciar, en niveles por encima de 10 mil puntos se genera una fuerte señal de compra, con implicaciones de ruptura muy alcista. Los objetivos se tendrían en 11,600 puntos. Recomendación: COMPRAR ENCIMA DE LOS 10000 PUNTOS. OBJETIVO 11.600 PUNTOS.

Pero, al margen de estos análisis también se puede utilizar estadísticos básicos, para ello es de utilidad el uso de un paquete estadístico tal como es el SPSS o el MINITAB, que por sus bondades tecnológicas logran obtener resultados que son de suma utilidad para la toma de decisiones en el mercado bursátil global, lo demostraremos con un ejemplo.

\section{ANALISIS CUANTATIVO DE MERCADOS BURSATILES GLOBALES}

1.1. Rentabilidad diaria del Dow Jones y el EuroStoXX: 2009

\section{Cuadro $\mathrm{N}^{\circ} 1$ : índices y rentabilidad del Dow Jones y EuroStoXX}

\begin{tabular}{|c|c|c|c|c|}
\hline $\begin{array}{l}\text { FECHA DE } \\
\text { NEGOCIACION }\end{array}$ & DOW JONES & $\begin{array}{c}\text { RENT ABILIDAD } \\
\text { D.J } \\
\end{array}$ & EUR STOXX & $\begin{array}{l}\text { RENT ABILIDAD } \\
\text { EUROST OXX }\end{array}$ \\
\hline $02 / 07 / 2009$ & 8280.74 & & 2369.65 & \\
\hline 01/07/2009 & 8504.06 & $-2.626 \%$ & 2449.73 & $-3.269 \%$ \\
\hline $30 / 06 / 2009$ & 8447 & $0.676 \%$ & 2401.69 & $2.000 \%$ \\
\hline $29 / 06 / 2009$ & 8529.38 & $-0.966 \%$ & 2437.72 & $-1.478 \%$ \\
\hline $26 / 06 / 2009$ & 8438.39 & $1.078 \%$ & 2389.91 & $2.000 \%$ \\
\hline $25 / 06 / 2009$ & 8472.4 & $-0.401 \%$ & 2402.32 & $-0.517 \%$ \\
\hline $24 / 06 / 2009$ & 8299.86 & $2.079 \%$ & 2418.46 & $-0.667 \%$ \\
\hline $23 / 06 / 2009$ & 8322.91 & $-0.277 \%$ & 2353.48 & $2.761 \%$ \\
\hline $22 / 06 / 2009$ & 8339.01 & $-0.193 \%$ & 2359.28 & $-0.246 \%$ \\
\hline $19 / 06 / 2009$ & 8539.73 & $-2.350 \%$ & 2434.77 & $-3.100 \%$ \\
\hline $18 / 06 / 2009$ & 8555.6 & $-0.185 \%$ & 2414.44 & $0.842 \%$ \\
\hline $17 / 06 / 2009$ & 8497.18 & $0.688 \%$ & 2383.71 & $1.289 \%$ \\
\hline $16 / 06 / 2009$ & 8504.67 & $-0.088 \%$ & 2425.97 & $-1.742 \%$ \\
\hline $15 / 06 / 2009$ & 8612.13 & $-1.248 \%$ & 2431.4 & $-0.223 \%$ \\
\hline $12 / 06 / 2009$ & 8799.26 & $-2.127 \%$ & 2509.22 & $-3.101 \%$ \\
\hline $11 / 06 / 2009$ & 8770.92 & $0.323 \%$ & 2522.33 & $-0.520 \%$ \\
\hline \multicolumn{5}{|l|}{$\begin{array}{c}\text { Volatilidad ( } \\
\text { Desviación Típica) }\end{array}$} \\
\hline \multicolumn{5}{|l|}{$\begin{array}{l}\text { Grado de } \\
\text { correlación }\end{array}$} \\
\hline $\begin{array}{c}\text { Rentabilidad } \\
\text { promedio diario del } \\
\text { mercado (RM) }\end{array}$ & $-0.375 \%$ & & & $-0.398 \%$ \\
\hline
\end{tabular}




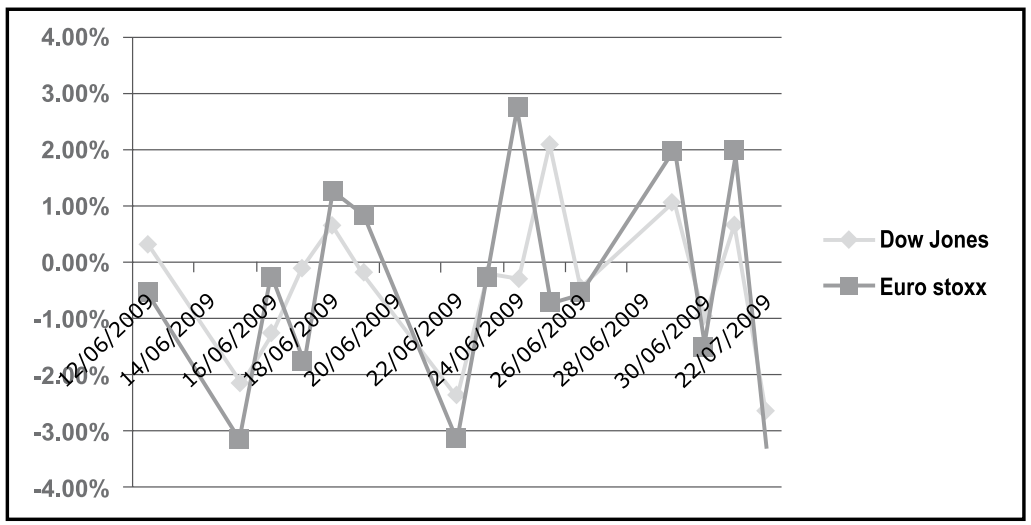

\section{ANALISIS DE LOS RESULTADOS:}

Por los resultados observados, el mercado bursátil de la zona del Euro presenta un mayor grado de volatilidad ya que cuenta con una mayor desviación típica con relación al Índice Dow Jones. Además los dos mercados presentan señales de eleva correlación el cual llega a $0.69(69 \%)$ es por ello que en el grafico $\mathrm{N}^{\circ} 2$ se observa que la rentabilidad de ambas Bolsas siguen la misma tendencia. La evidencia empírica

Cuadro N². Rentabilidad diaria de acciones globales: 2009

\begin{tabular}{|l|l|l|l|l|l|l|l|l}
\hline & CHEVRON & RENTABILIDAD & PFIZER & RENTABILIDAD & BAYER & RENTABILIDAD & PHILIPS & RENTABILIDAD \\
\hline $02 / 07 / 2009$ & 64.42 & & 14.48 & & 37.38 & & 12.74 & \\
\hline $01 / 07 / 2009$ & 66.52 & $-3.157 \%$ & 14.9 & $-2.819 \%$ & 38.72 & $-3.461 \%$ & 13.22 & $-3.631 \%$ \\
\hline $30 / 06 / 2009$ & 66.25 & $0.408 \%$ & 15 & $-0.667 \%$ & 38.22 & $1.308 \%$ & 13.13 & $0.685 \%$ \\
\hline $29 / 06 / 2009$ & 66.88 & $-0.942 \%$ & 15.26 & $-1.704 \%$ & 38.53 & $-0.805 \%$ & 13.28 & $-1.130 \%$ \\
\hline $26 / 06 / 2009$ & 65.95 & $1.410 \%$ & 15.19 & $0.461 \%$ & 38.55 & $-0.052 \%$ & 12.99 & $2.232 \%$ \\
\hline $25 / 06 / 2009$ & 66.87 & $-1.376 \%$ & 15.33 & $-0.913 \%$ & 38.66 & $-0.285 \%$ & 12.75 & $1.882 \%$ \\
\hline $24 / 06 / 2009$ & 65.72 & $1.750 \%$ & 14.72 & $4.144 \%$ & 38.88 & $-0.566 \%$ & 12.89 & $-1.086 \%$ \\
\hline $23 / 06 / 2009$ & 65.96 & $-0.364 \%$ & 14.73 & $-0.068 \%$ & 38.46 & $1.092 \%$ & 12.64 & $1.978 \%$ \\
\hline $22 / 06 / 2009$ & 65.76 & $0.304 \%$ & 14.79 & $-0.406 \%$ & 38.95 & $-1.258 \%$ & 12.72 & $0.629 \%$ \\
\hline $19 / 06 / 2009$ & 68.06 & $-3.379 \%$ & 15 & $-1.400 \%$ & 39.99 & $-2.601 \%$ & 13.14 & $-3.196 \%$ \\
\hline $18 / 06 / 2009$ & 68.43 & $-0.541 \%$ & 14.92 & $0.536 \%$ & 39.98 & $0.025 \%$ & 13.05 & $0.690 \%$ \\
\hline $17 / 06 / 2009$ & 68.83 & $-0.581 \%$ & 14.58 & $2.332 \%$ & 39.36 & $1.575 \%$ & 12.99 & $0.462 \%$ \\
\hline $16 / 06 / 2009$ & 69.88 & $-1.503 \%$ & 14.16 & $2.966 \%$ & 40.05 & $-1.723 \%$ & 13.24 & $-1.888 \%$ \\
\hline $15 / 06 / 2009$ & 71.08 & $-1.688 \%$ & 14.13 & $0.212 \%$ & 39.82 & $0.578 \%$ & 13.33 & $0.675 \%$ \\
\hline $12 / 06 / 2009$ & 72.67 & $-2.188 \%$ & 14.76 & $-4.268 \%$ & 41.54 & $-4.141 \%$ & 13.67 & $2.487 \%$ \\
\hline $11 / 06 / 2009$ & 71.9 & $1.071 \%$ & 14.63 & $0.889 \%$ & 41.9 & $-0.859 \%$ & 13.75 & $0.582 \%$ \\
\hline
\end{tabular}

FUENTE; WWW.FINANZAS.COM

ELABORACION PROPIA 
señala que la bolsa norteamericana marca tendencias de todas las bolsas del mundo, de allí la explicación sl comportamiento simétrico de los mercados bursátiles globales. Dada la característica correlativa de estos dos mercados, para un inversionistas le podría representar una buena oportunidad para obtener buenos resultados por sus inversiones, o en lo contrario también podría obtener significativas pérdidas.

Por la crisis financiera global el rendimiento promedio esperado diario para ambas bolsas es negativo, situación que se profundiza en la Zona del Euro, cuya tasa llega al - 0.398\%. El riesgo se complementa por dos lados, por la desviación típica y por el coeficiente de correlación. Como señalan los textos de finanzas, si un inversionista desea cobertura ante riesgos, tiene que poseer en su portafolio activos financieros cuyo coeficiente de correlación sea negativo.

2.2. Rentabilidad de acciones globales: Chevron, Phizer, Bayer, Philip

Gráfico N 2: Rentabilidad diaria de las acciones globales: 2009

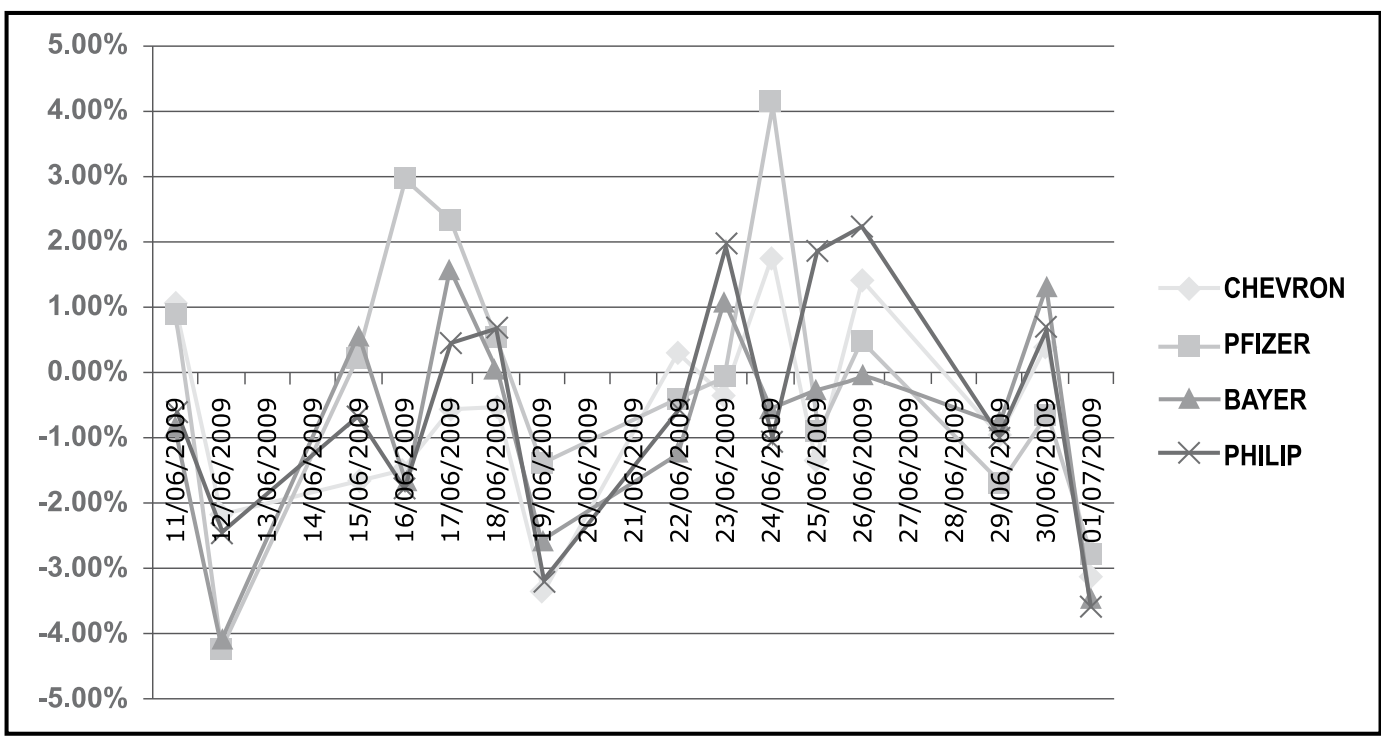

\section{RESULTADOS DEL ANALISIS}

a. Determinación del Beta de cada acción y desviación típica(riesgos)

BETA DE CHEVRON: $\frac{\text { COVARIANZA (CHEVRON,DOWJONES ) }}{\text { VARIANZA DE DOW JONES }}=\frac{\operatorname{Cov}(\mathrm{Rm} ; \mathrm{Ri})}{\operatorname{Var}(\mathrm{Rm})}$

$0.0173 \% / 0174 \%=1.007100997=\mathbf{1 . 0}$

BETA DE BAYER $=\frac{\text { COVARIANZA }(\text { BAYER,EUR STOXX })}{\text { VARIANZA DE EUROSTOXX }}=\frac{\operatorname{Cov}(\mathrm{Rm} ; \mathrm{Ri})}{\operatorname{Var}(\mathrm{Rm})}$

$0.027 \% / 0.037 \%=1.007100997=\mathbf{0 . 7 3 2 5}$

Resumen de las Betas y las desviaciones típicas de cada título global

\begin{tabular}{|l|c|c|}
\hline ACCION & $\begin{array}{l}\text { BETA DE CADA } \\
\text { ACCION }\end{array}$ & DESVIACON TIPICA \\
\hline CHEVRON & 1,00 & $1,548 \%$ \\
\hline PZIFER & $\mathbf{1 . 2 2}$ & $\mathbf{2 . 1 5 8 \%}$ \\
\hline BAYER & 0.73 & $1.682 \%$ \\
\hline PHILIP & 0.80 & $1,828 \%$ \\
\hline
\end{tabular}




\section{ANALISIS DE LOS RESULTADOS}

De las cuatro acciones globales analizadas las más riesgosa es la Phizer, afirmación que queda demostrada con el resultado del Beta y la desviación típica que llega a 1.22 y $2.158 \%$ respectivamente. En contraste al primer resultado, se tiene a Chevron cuyo Beta nos dice que el rendimiento que pueda lograr esta acción será igual al rendimiento que alcance el EurostoXX, asimismo este título muestra menor indicador de volatilidad, ya que su desviación típica solo llega a $1,548 \%$.

Tomado en cuenta el Beta, la acción que presenta menor nivel de riesgo es el de Bayer que llega solo a 0.73 . Hay que tener en cuenta que todo titulo cuyo Beta sea menor 1 son tipificadas como de bajo riesgo y los que superen a 1 son los de elevado riesgo. Ahora si el inversionista desea estructurar un portafolio podría, adquirir títulos de altas y bajas betas, con lo cual quedaría coberturado ante cambios cíclicos del mercado.

Para complementar el análisis es necesario realizar la prueba de Pearson o correlación, a fin de determinar que acciones están directa e inversamente relacionadas, los resultados se puede determinar utilizando el paquete estadístico SPSS-16. El marco teórico nos dice, si la correlación es alta y directa los activos demandados son de alto riesgo, de lo contrario si la correlación es inversa y se acerca a -1 , el inversionista tendrá una cuasi perfecta cobertura ante riesgos que se pueda presentar en el mercado.

Si su propósito es estructurar una cartera con Phizer y Bayer, es decir participar en el Índice Dow Jones y la segunda al Euro stoXX el riesgo que incurriría sería el siguiente:

- Coeficiente de correlación (Pfizer, Bayer) : r : 0.54

- Desviación típica de Pfizer : 2.158\%

- Desviación típica de Bayer : $1.682 \%$

- Participación de Pfizer en el portafolio: $40 \%$

- Participación de de Bayer en el portafolio: $60 \%$

$\mathrm{Al}$ operativizar estos resultados el riesgo de la cartera seria igual a : $1.6430 \%$

Y el beta del portafolio compuesto por Pfizer y Bayer es:

Bc: $1.22 X 0.40+0.73 \times 0.60: \mathbf{0 . 9 2 6}$

\section{CONCLUSIONES}

- La crisis financiera global presenta señales de ser un problema de carácter estructural, por lo que medidas de tipo monetarias como fiscales tradicionales, no lograrán revertir la incertidumbre que se presenta en los mercados.
- La volatilidad de los mercados financieros, y su situación de crisis estructural, obliga a los inversionistas particulares e institucionales, a tener una cartera de títulos más diversificada, Lo cual implica demandar activos financieros globales.

- La globalización de los mercados abre oportunidades para lograr una mayor y mejor diversificación del portafolio de un inversionista, con lo cual se lograría una mayor cobertura o en todo caso mejores rendimientos esperados.

- Los mercados bursátiles globales, como el de Estados Unidos o la Zona del Euro, son espacios de oportunidades para que los inversionistas institucionales logren estructurar portafolios con acciones emitidas por las empresas más importantes del mundo.

\section{REFERENCIAS BIBLIOGRÁFICAS}

1. Brigham, Hosuton ( 2005). "Fundamentos de Administración Financiera”, Thomson Editores. México

2. Elbaun, Marcelo( 2004). "Administración de carteras de inversión", 1 $1 \mathrm{ra}$ ed. Buenos Aires, Ediciones Machi.

3. Francis, J.C(1971). "Análisis y gestión de cartera de valores", 1 ra ed. Madrid.

4. Mendenhall, William; Scheaffer, Richard L. y Dennis D. Wackerly (1994) Estadística Matemática con Aplicaciones. México: Grupo Editorial Iberoamérica. 2da. Edición.

5. www.finanzas.com

6. www.bolsamania.com

7. www.wilkipedia.com 